\title{
Subject Status Character Result in Standard Format
}

National Cancer Institute

\section{Source}

National Cancer Institute. Subject Status Character Result in Standard Format. NCI

Thesaurus. Code C117664.

The standard character or string for representation and reporting of subject status data. 